\title{
Atomic Resolution Scanning Transmission Electron Microscopy of Two-Dimensional Layered Transition Metal Dichalcogenides
}

\author{
Ning Lu, Jinguo Wang, Juan Pablo Oviedo, Guoda Lian, Moon Jea Kim* \\ Department of Materials Science and Engineering, The University of Texas at Dallas, Richardson, TX 75080, USA
}

*Correspondence to:
Kim MJ,
Tel: +1-972-883-6635
Fax: +1-972-883-5725
E-mail: moonkim@utdallas.edu

Received September 21, 2015

Revised September 30, 2015

Accepted September 30, 2015

\begin{abstract}
Transition metal dichalcogenides (TMDs) are a class of two-dimensional (2D) materials that have attracted growing interest because of their promising applications. The properties of TMDs strongly depend on the crystalline structure and the number and stacking sequence of layers in their crystals and thin films. Though electrical, mechanical, and magnetic studies of 2D materials are being conducted, there is an evident lack of direct atom-by-atom visualization, limiting insight on these highly exciting material systems. Herein, we present our recent studies on the characterization of $2 \mathrm{D}$ layered materials by means of aberration corrected scanning transmission electron microscopy (STEM), in particular via high angle annular dark field (HAADF) imaging. We have identified the atomic arrangements and defects in $2 \mathrm{H}$ stacked TMDs, $1 \mathrm{~T}$ stacked TMDs, distorted $1 \mathrm{~T}$ stacked TMDs, and vertically integrated heterojunctions of 2D TMDs crystals.
\end{abstract}

Key Words: Aberration correction, Scanning transmission electron microscopy, Twodimension, Transition metal dichalcogenides

\section{INTRODUCTION}

It is well documented that the dimensionality of the material plays a crucial role in determining its fundamental properties in addition to the composition and arrangement of atoms. One of the most researched two-dimensional (2D) material is graphene, which exhibits exotic condensedmatter phenomena that are absent in bulk graphite (Castro Neto et al., 2009). Research in graphene and the developed methodology of preparing ultrathin layers have led to the exploration of other 2D materials (Wang et al., 2012). In particular, single layers of transition metal dichalcogenides (TMDs) with lamellar structures similar to that of graphite have drawn significant attention because some of them are semiconductors with sizable band gaps and are naturally abundant (Wang et al., 2012). TMDs form as $\mathrm{MX}_{2}$, where $\mathrm{M}$ is a transition metal (Mo, W, Hf, etc.) and $\mathrm{X}$ is a chalcogen ( $\mathrm{S}, \mathrm{Se}$, and $\mathrm{Te}$ ). Recent reports have explored the crystal structures, synthesis, and electronic properties of TMDs using both experimental and theoretical techniques (Chhowalla et al., 2013). These studies suggest that the absence of dangling bonds (Jena, 2013) and the wide range of band gaps (from $3.5 \mathrm{eV}$ to $<1 \mathrm{eV}$ ) (Gong et al., 2013) make TMDs relevant in practical devices, such as field effect transistors (Radisavljevic et al., 2011; Ilatikhameneh et al., 2015) and photo-sensors (Yin et al., 2012; Perea-Lopez et al., 2013).

TMDs exhibit diverse properties that depend on their composition and can behave as semiconductors (e.g., $\mathrm{MoS}_{2}$, $\mathrm{WS}_{2}$ ), semimetals (e.g., $\mathrm{WTe}_{2}$, TiSe $\mathrm{T}_{2}$ ), true metals (e.g., $\mathrm{NbS}_{2}$, $\mathrm{VSe}_{2}$ ), and superconductors (e.g., $\mathrm{NbSe}_{2}, \mathrm{TaS}_{2}$ ) (Wilson \& Yoffe, 1969). TMDs have layered structures with an X-M-X atomic stacking sequence in each layer. There exist three main structures in TMDs: $2 \mathrm{H}, 1 \mathrm{~T}$, and distorted $1 \mathrm{~T}$ ( $\mathrm{Td}$, also known as $\left.1 \mathrm{~T}^{\prime}\right)$. The properties of TMDs strongly depend on the crystalline structure and the number and stacking sequence of layers in their crystals and thin films (Wang et

This work was supported by the Center for Low Energy Systems Technology (LEAST), one of six centers supported by the STARnet phase of the Focus Center Research Program (FCRP), a Semiconductor Research Corporation program sponsored by MARCO and DARPA, and by the Louis Beecherl, Jr. endowment funds.

@ This is an open-access article distributed under the terms of the Creative Commons Attribution Non-Commercial License (http://creativecommons.org/licenses/by-nc/4.0) which permits unrestricted noncommercial use, distribution, and reproduction in any medium, provided the original work is properly cited.

Copyrights @ 2015 by Korean Society of Microscopy 
al., 2012). For example, bulk $\mathrm{MoS}_{2}$ has an indirect band gap of $1.29 \mathrm{eV}$. The indirect band gap shifts up by more than 0.6 $\mathrm{eV}$ by decreasing the number of layers. A monolayer $\mathrm{MoS}_{2}$ eventually becomes a direct band gap semiconductor (Mak et al., 2010). Different from materials grown via traditional hetero-epitaxy, 2D materials can achieve hetero-epitaxy by van der Waals (vdW) force between layers without consideration of the lattice mismatch. Although electrical studies, magnetic studies, mechanical studies, and/or any combination thereof of $2 \mathrm{D}$ materials as new material systems have been conducted, there is a lack of direct atom-by-atom visualizations, limiting our understanding of these highly exciting material systems. Herein, we present our recent studies on the characterization of 2D layered materials by means of aberration corrected scanning transmission electron microscopy (STEM), in particular via high angle annular dark field (HAADF) imaging.

\section{H STRUCTURAL TMDs}

Typical TMDs $\mathrm{MX}_{2}$ materials with $2 \mathrm{H}$ structure are $\mathrm{MoS}_{2}$, $\mathrm{MoSe}_{2}$, and $\mathrm{WSe}_{2}$. When the $\mathrm{M}$ atoms are coordinated by a trigonal prism of $\mathrm{X}$ atoms, the structure is defined as the $2 \mathrm{H}$ phase (Wilson \& Yoffe, 1969). There are two X-M-X layers in each unit cell of the $2 \mathrm{H}$ structure. Group VIb TMDs that adopt the $2 \mathrm{H}$ structure, such as $\mathrm{MoS}_{2}$ and $\mathrm{WS}_{2}$, are usually semiconductors with band gaps between 1 to $2 \mathrm{eV}$ (Wang et al., 2012; Gong et al., 2013). Firstly, we are able to identify the atomic nature of the single layer $2 \mathrm{H}$ TMDs in planeview by HAADF-STEM imaging technique. A good example is shown in Fig. 1A, a HAADF-STEM image of the plane view of monolayer $2 \mathrm{H}-\mathrm{MoS}_{2}$ (Oviedo et al., 2015). Contrast in a HAADF-STEM image is approximately proportional to $\mathrm{Z}^{2}$, where $\mathrm{Z}$ is the atomic number (Hartel et al., 1996). This provides a way to identify different atomic species by Z-contrast imaging. Using HAADF, molybdenum and sulfur atomic columns are distinguishable from one another (Fig. 1A). The HAADF image reveals that the molybdenum and sulfur atoms are arranged in a hexagonal configuration with Mo-S and Mo-Mo separations of $\sim 0.19$ and $\sim 0.33 \mathrm{~nm}$, respectively, coinciding with the structure of $2 \mathrm{H}-\mathrm{MoS}_{2}$ (Fig. $1 \mathrm{~B})$. Fig. $1 \mathrm{~B}$ and $\mathrm{C}$ show the schematic model of $2 \mathrm{H}-\mathrm{MX}_{2}$ in [0001] and [11 $\overline{2} 0]$, respectively. It is worth noting that a Mo atom substitutes an $\mathrm{S}$ atom at the edge of the monolayer flake, indicated by the red arrow in Fig. 1A. Z-contrast imaging combined with energy dispersive X-ray spectroscopy (EDS) was used to investigate the distribution of doped manganese in a $\mathrm{MoS}_{2}$ monolayer (Zhang et al., 2015). Compared to Mo, the Mn atoms are expected to have $~ 50 \%$ intensity in HAADF. It is revealed that Mo is $~ 2 \%$ substituted by $\mathrm{Mn}$ atoms and $\mathrm{Mn}$ is not segregated to domain boundaries.

Cross section structures of $2 \mathrm{H}-\mathrm{MoS}_{2}$ grown via chemical vapor deposition (CVD) on graphene and $\mathrm{SiC}$ was also investigated by high resolution TEM and HAADF. It is found that the $\mathrm{MoS}_{2}$ layer appears to be "blind" to thickness variations in the underlying graphene when there are no defects in the top layer of the graphene. However, when the top graphene layer is interrupted, the $\mathrm{MoS}_{2}$ will also be discontinuous. Apparently, in the case where additional layers of graphene are formed in a manner as to maintain a flat surface, the graphene appears to shield the $2 \mathrm{H}-\mathrm{MoS}_{2}$ from the influence of the SiC surface. On the other hand, when defective graphene is present, multilayered $\mathrm{MoS}_{2}$ is almost always present (Lin et al., 2014).

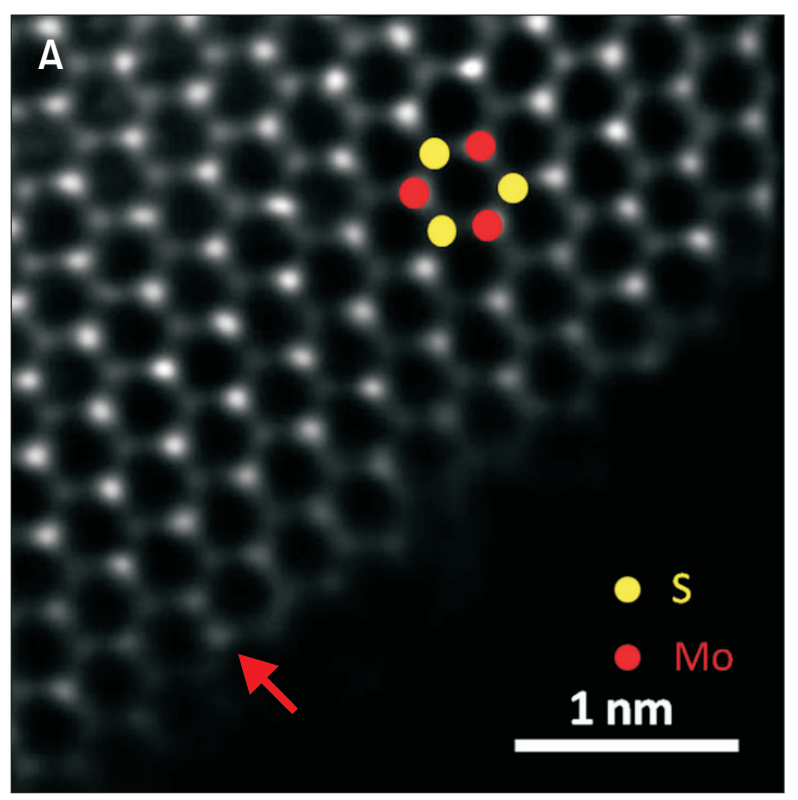

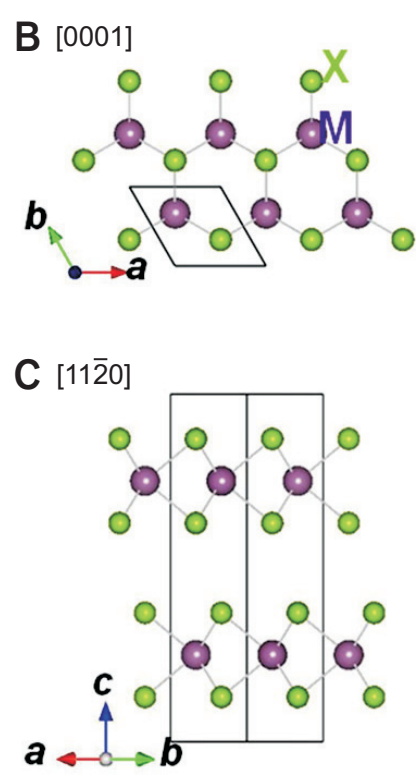

Fig. 1. (A) High angle annular dark field-scanning transmission electron microscopy image of the plane view of monolayer $2 \mathrm{H}-\mathrm{MoS}_{2}$. Mo atom substitutes an $\mathrm{S}$ atom at the edge of the monolayer, as indicated by the red arrow. (B, C) Schematic model of $2 \mathrm{H}-\mathrm{MX}_{2}$ in [0001] and [11 $\overline{2} 0]$, respectively. a, b, and c indicate the unit cell axis. 


\section{T STRUCTURAL TMDs}

Typical TMDs $\mathrm{MX}_{2}$ materials with $1 \mathrm{~T}$ structure are $\mathrm{SnS}_{2}$, $\mathrm{SnSe}_{2}, \mathrm{HfS}_{2}$, and $\mathrm{HfSe}_{2}$. When one X layer is shifted such that the metal becomes octahedrally coordinated, the structure is referred to as the $1 \mathrm{~T}$ phase (Wilson \& Yoffe, 1969). In this case, there is only one X-M-X layer in the unit cell. 1T structure TMDs cover a broad spectrum of electrical properties; insulators such as $\mathrm{HfS}_{2}$, semiconductors such as $\mathrm{HfSe}_{2}$, and metals such as $\mathrm{TiS}_{2}$ (Wilson \& Yoffe, 1969). The atomic nature of single layer $1 \mathrm{~T}$ TMDs was also investigated by HAADFSTEM in plane-view. Fig. 2A shows a HAADF-STEM image of the plane view of $1 \mathrm{~T}-\mathrm{HfSe}_{2}$. The atomic columns of hafnium and selenium are easily distinguishable from one another. $1 \mathrm{~T}-\mathrm{MX}_{2}$ has different features compared with $2 \mathrm{H}-$ $\mathrm{MX}_{2}$, which shows $\mathrm{X}$ hexagonal pattern with an $\mathrm{M}$ center (Fig. $2 \mathrm{~A}$ and $\mathrm{B})$. Fig. $2 \mathrm{~B}$ and $\mathrm{C}$ show a schematic model of $1 \mathrm{~T}-\mathrm{MX}_{2}$ in [001] and [110], respectively. In the cross sectional view along the same projection direction, [110] in $1 \mathrm{~T}$ (Fig. 2C) and $[11 \overline{2} 0]$ in $2 \mathrm{H}$ (Fig. 1C) structures show different features, which can be used to identify the phase in side view imaging. $1 \mathrm{~T}-\mathrm{HfSe}_{2}$ is predicted to have a large electron affinity and a small band gap, which can be used as the drain in vertically stacked, "broken-gap" band alignment tunnel field-effect transistors (Yue et al., 2015). $\mathrm{HfSe}_{2}$ was grown by molecular beam epitaxy (MBE) on different substrates, such as highly

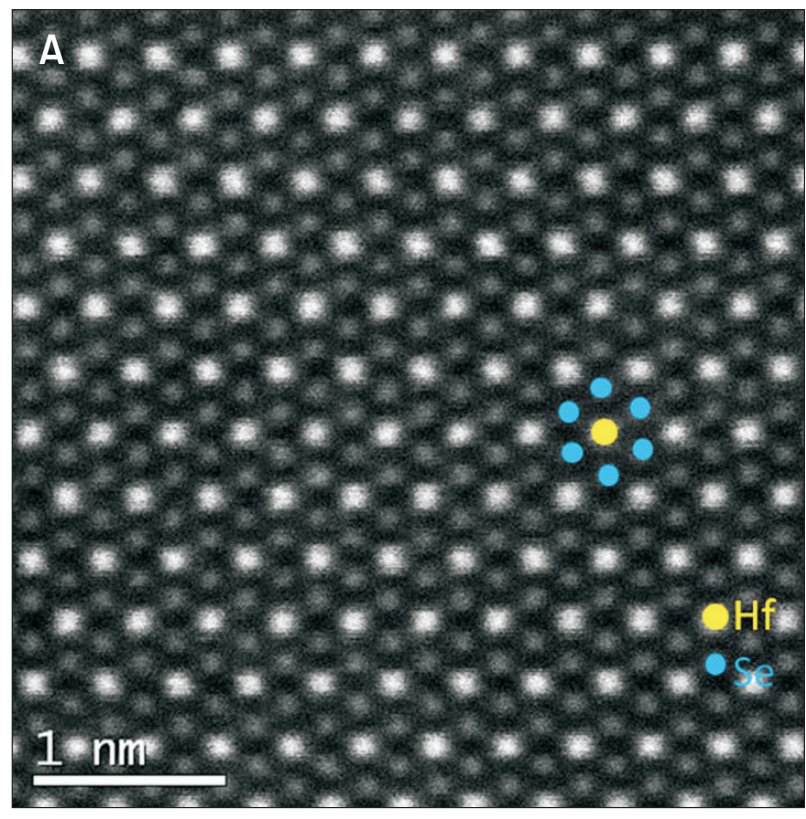

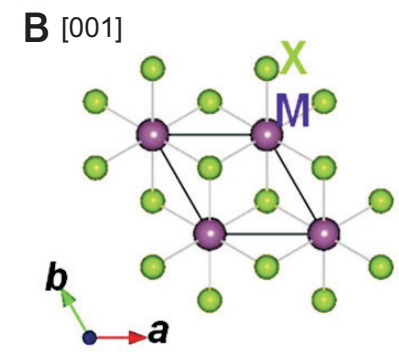

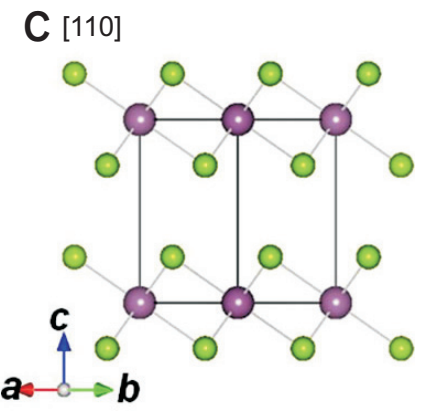

Fig. 2. (A) High angle annular dark field-scanning transmission electron microscopy image of the plane view of 1T-HfSe $e_{2}$ (B, C) Schematic model of 1T$\mathrm{MX}_{2}$ in [001] and [110], respectively. a, b, and $\mathrm{c}$ indicated the unit cell axis.
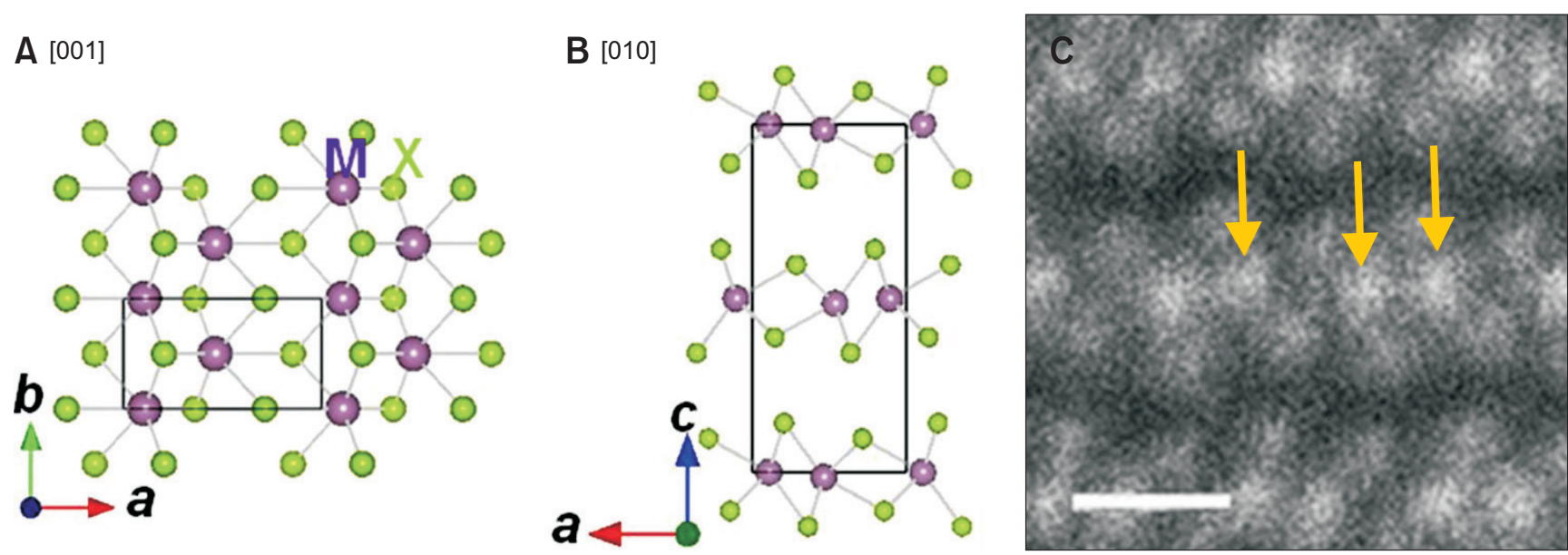

Fig. 3. Schematic model of Td-MX in [001] (A) and [010] (B), respectively. a, b, and c indicated the unit cell axis. (C) Cross-sectional high angle annular dark field-scanning transmission electron microscopy image of the $\mathrm{Td}-\mathrm{WTe}_{2}$ in [010] zone axis orientation. Arrows indicate the vertical shift of Mo atoms along the $\mathrm{c}$ axis. Scale bar $=0.5 \mathrm{~nm}$. 
ordered pyrolytic graphite (HOPG), $\mathrm{MoS}_{2}, \mathrm{Al}_{2} \mathrm{O}_{3}$, and $\mathrm{SiO}_{2} /$ Si. On HOPG and $\mathrm{MoS}_{2}$, multilayered crystalline $\mathrm{HfSe}_{2}$ was grown without misfit dislocations. However, on $\mathrm{Al}_{2} \mathrm{O}_{3}$ and $\mathrm{SiO}_{2} / \mathrm{Si}$, the grown multilayered $\mathrm{HfSe}_{2}$ is of poor quality. It is found that the top layer of MBE grown $1 \mathrm{~T}-\mathrm{HfSe}_{2}$ is easily oxidized upon exposure to air. A Si capping layer can be used to minimize surface oxidation, which is confirmed by electron energy loss spectroscopy (EELS) analysis (Yue et al., 2015).

\section{DISTORTED 1T STRUCTURAL TMDs}

Most TMDs exist in the $2 \mathrm{H}$ or $1 \mathrm{~T}$ structures at ambient conditions. However, there exists another structure called distorted $1 \mathrm{~T}$ structure. $\mathrm{WTe}_{2}$ and $\beta$-MoTe $\mathrm{M}_{2}$ adopts the distorted 1T structure with semi-metallic conduction (Dawson \& Bullett, 1987). Unlike the $1 \mathrm{~T}$ and 2H structures, Td-MX $\mathrm{X}_{2}$ contain non-coplanar atomic layers of both $\mathrm{M}$ and $\mathrm{X}$ (Fig. 3B). This puckering of layers is caused by an additional structural distortion: $\mathrm{M}$ metallic chains are formed within the $\mathrm{X}$ chalcogen layers along the $\mathrm{b}$ axis of the orthorhombic unit cell (Fig. 3A). We have successfully imaged the Td-WTe ${ }_{2}$ from three major crystalline orientations ([100], [010], and [001]) and determined the atomic species and their positions in this crystal. The vertical shift of $\mathrm{W}$ and Te atoms along the $\mathrm{c}$ axis can be easily viewed in the [010] orientation (Fig. 3C). Both $\mathrm{W}$ and Te atoms form zigzag-like planes. Combined with first principal calculation, the characteristic atomic and electronic properties of the material were investigated. Results showed the band structure characteristics of $\mathrm{Td} \mathrm{WTe}_{2}$ to be semimetallic.

\section{VERTICAL 2D VAN DER WAALS TMDS HETEROJUNCTIONS}

Vertical integration of $2 \mathrm{D}$ materials by $\mathrm{vdW}$ is predicted to lead to novel electronic and optical properties not found in the individual constituent layers. The interface and defects of various heterojunctions of TMDs, such as CVD grown $\mathrm{MoS}_{2}$ on graphene/SiC (Lin et al., 2014) and MBE grown $\mathrm{HfSe}_{2}$ on $\mathrm{MoS}_{2}$ and HOPG (Yue et al., 2015), have been investigated by HAADF-STEM at the atomic scale. Here, we present the STEM study of another more complicated vertical heterojunction with 2 types of TMDs and graphene: $\mathrm{MoS}_{2}$, $\mathrm{WSe}_{2}$, and graphene. The n-type TMDs $\left(\mathrm{MoS}_{2}\right)$ act as barrier layers that electronically confine the p-type TMD ( $\left.\mathrm{WSe}_{2}\right)$, leading to resonant tunneling transport of carriers. $\mathrm{MoS}_{2} /$ $\mathrm{WSe}_{2} /$ graphene vertical junction begins with the synthesis of epitaxial graphene from $\mathrm{SiC}$ followed by vapor transport or MOCVD of $\mathrm{WSe}_{2}$ and vapor transport of $\mathrm{MoS}_{2}$ (Lin et al., 2015).

Fig. 4 shows HAADF and annular bright field (ABF) images of the four-layer $\mathrm{MoS}_{2}$ /bi-layer WSe $\mathrm{W}_{2}$, tri-layer graphene, and the $\mathrm{SiC}$ substrate. Using $\mathrm{HAADF}$, the $\mathrm{MoS}_{2}$ and $\mathrm{WSe}_{2}$ layers are clearly distinguishable (Fig. $4 \mathrm{~A}$ ). The bi-layer $\mathrm{WSe}_{2}$ has the brightest contrast and the four-layer $\mathrm{MoS}_{2}$ has the second brightest contrast. However, as this sample is composed of both heavy and light elements (carbon), the carbon's relatively lacking contrast makes it difficult to reveal graphene's layer structure. The recently developed ABF imaging technique enables simultaneous visualization of light and heavy elements (Okunishi et al., 2009). Fig. 4B shows the ABF image from the same area with HAADF. The contrast of $\mathrm{ABF}$ is reversed compared with HAADF. $\mathrm{MoS}_{2}$ and $\mathrm{WSe}_{2}$ layers appeared as dark lines and three graphene layers are clearly visible on top of the $\mathrm{SiC}$ substrate. Thus, the $\mathrm{MoS}_{2} / \mathrm{WSe}_{2} /$ graphene layer structure can be obviously revealed by HAADF and ABF imaging. STEM imaging combined with EDS and EELS line scans verified the heterojunction is not a manifestation of the alloying of the constituent TMDs, but indeed are unique layers with pristine interfaces with atomic precision. It is also noted that defects and edge states in the base 2D layer lead to low energy nucleation sites, and therefore multilayer growth
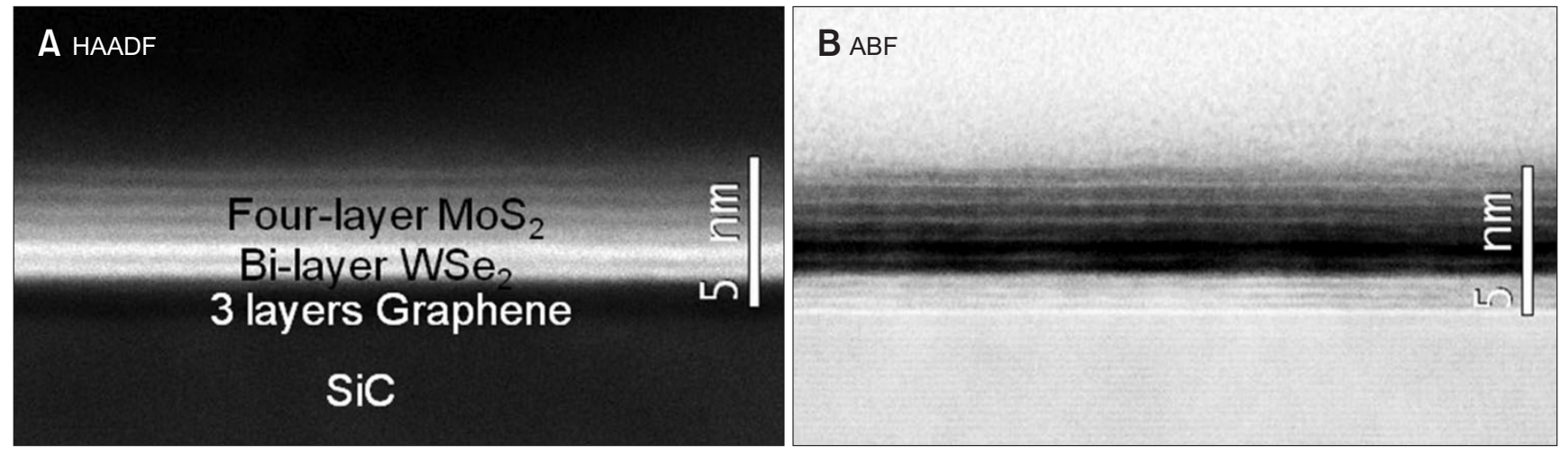

Fig. 4. High angle annular dark field (HAADF) (A) and annular bright field (ABF) (B) imaging of $\mathrm{MoS}_{2} / \mathrm{WSe}_{2} /$ graphene two-dimensional heterojunction on $\mathrm{SiC}$ substrate. In Fig. 4A, HAADF image clearly distinguishes the difference between bi-layer $\mathrm{WSe}_{2}$ and four-layer $\mathrm{MoS}_{2}$. ABF image (B) enhances the contrast of the tri-layer graphene. 
of a top layer is highly probable at these regions (Lin et al., 2015). These results demonstrate the feasibility and significant potential of fabricating 2D material based heterojunctions with tunable band alignments for a variety of nanoelectronic and optoelectronic applications.

\section{CONCLUSIONS}

TMDs as re-discovered materials have rapidly moved to the forefront of "next generation" materials with many applications. We reviewed our recent studies on the characterization of $2 \mathrm{D}$ layered materials by means of aberration corrected
STEM, in particular via HAADF imaging combined with EDS and EELS analysis. The location and nature of individual atoms, defects, and vertical heterojunctions of the 2D TMDs materials with $2 \mathrm{H}, 1 \mathrm{~T}$, and Td structures have been discussed. With the advantages of aberration corrected STEM, direct atom-by-atom visualization is expanding our understanding of these highly exciting material systems and field of research.

\section{CONFLICT OF INTEREST}

No potential conflict of interest relevant to this article was reported.

\section{REFERENCES}

Castro Neto A H, Guinea F, Peres N M R, Novoselov K S, and Geim A K (2009) The electronic properties of graphene. Rev. Mod. Phys. 81, 109-162.

Chhowalla M, Shin H S, Eda G, Li L J, Loh K P, and Zhang H (2013) The chemistry of two-dimensional layered transition metal dichalcogenide nanosheets. Nat. Chem. 5, 263-275.

Dawson W G and Bullett D W (1987) Electronic-structure and crystallography of $\mathrm{MoTe}_{2}$ and $\mathrm{WTe}_{2}$. J. Phys. C Solid State 20, 6159-6174.

Gong C, Zhang H J, Wang W H, Colombo L, Wallace R M, and Cho K $J$ (2013) Band alignment of two-dimensional transition metal dichalcogenides: application in tunnel field effect transistors. Appl. Phys. Lett. 103, 053513.

Hartel P, Rose H, and Dinges C (1996) Conditions and reasons for incoherent imaging in STEM. Ultramicroscopy 63, 93-114.

Ilatikhameneh H, Tan Y, Novakovic B, Klimeck G, Rahman R, and Appenzeller J (2015) Tunnel field-effect transistors in 2D transition metal dichalcogenide materials. IEEE J. Explor. Solid-State Computat. Devices Circuits 1, 12-18.

Jena D (2013) Tunneling transistors based on graphene and 2-D crystals. Proc. IEEE 101, 1585-1602.

Lin Y C, Ghosh R K, Addou R, Lu N, Eichfeld S M, Zhu H, Li M Y, Peng X, Kim M J, Li L J, Wallace R M, Datta S, and Robinson J A (2015) Atomically thin resonant tunnel diodes built from synthetic van der Waals heterostructures. Nat. Commun. 6, 7311.

Lin Y C, Lu N, Perea-Lopez N, Li J, Lin Z, Peng X, Lee C H, Sun C, Calderin L, Browning P N, Bresnehan M S, Kim M J, Mayer T S, Terrones M, and Robinson J A (2014) Direct synthesis of van der Waals solids. ACS Nano 8, 3715-3723.

Mak K F, Lee C, Hone J, Shan J, and Heinz T F (2010) Atomically thin $\mathrm{MoS}_{2}$ : a new direct-gap semiconductor. Phys. Rev. Lett. 105, 136805.
Okunishi E, Ishikawa I, Sawada H, Hosokawa F, Hori M, and Kondo Y (2009) Visualization of light elements at ultrahigh resolution by STEM annular bright field microscopy. Microsc. Microanal. 15, 164-165.

Oviedo J P, Santosh K C, Lu N, Wang J G, Cho K, Wallace R M, and Kim M J (2015) In situ TEM characterization of shear-stress-induced interlayer sliding in the cross section view of molybdenum disulfide. ACS Nano 9, 1543-1551.

Perea-Lopez N, Elias A L, Berkdemir A, Castro-Beltran A, Gutierrez H R, Feng S M, Lv R T, Hayashi T, Lopez-Urias F, Ghosh S, Muchharla B, Talapatra S, Terrones H, and Terrones M (2013) Photosensor device

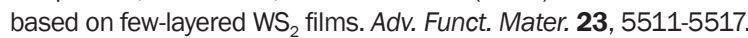

Radisavljevic B, Radenovic A, Brivio J, Giacometti V, and Kis A (2011) Single-layer $\mathrm{MoS}_{2}$ transistors. Nat. Nanotechnol. 6, 147-150.

Wang Q H, Kalantar-Zadeh K, Kis A, Coleman J N, and Strano M S (2012) Electronics and optoelectronics of two-dimensional transition metal dichalcogenides. Nat. Nanotechnol. 7, 699-712.

Wilson J A and Yoffe A D (1969) Transition metal dichalcogenides discussion and interpretation of observed optical, electrical and structural properties. Adv. Phys. 18, 193-335.

Yin Z Y, Li H, Li H, Jiang L, Shi Y M, Sun Y H, Lu G, Zhang Q, Chen X D, and Zhang $\mathrm{H}$ (2012) Single-layer $\mathrm{MoS}_{2}$ phototransistors. ACS Nano 6, 7480 .

Yue R Y, Barton A T, Zhu H, Azcatl A, Pena L F, Wang J, Peng X, Lu N, Cheng L X, Addou R, Mcdonnell S, Colombo L, Hsu J W P, Kim J, Kim M J, Wallace R M, and Hinkle C L (2015) $\mathrm{HfSe}_{2}$ thin films: 2D transition metal dichalcogenides grown by molecular beam epitaxy. ACS Nano 9, 474-480.

Zhang K, Feng S, Wang J, Azcatl A, Lu N, Addou R, Wang N, Zhou C, Lerach J, Bojan V, Kim M J, Chen L Q, Wallace R M, Terrones M, Zhu J, and Robinson J A (2015) Manganese doping of monolayer $\mathrm{MoS}_{2}$ : the substrate is critical. Nano Lett. doi: 10.1021/acs. nanolett.1025b02315. 